\title{
14. POST-MIDDLE OLIGOCENE EOLIAN DEPOSITION FROM THE TRADE WINDS OF THE SOUTHEAST PACIFIC1
}

\author{
Maurice K. Bloomstine and David K. Rea, Department of Atmospheric and Oceanic Science, \\ The University of Michigan ${ }^{2}$
}

\begin{abstract}
Eolian dust preserved in deep-sea sediments provides a record of atmospheric circulation throughout geologic time. This eolian component has been isolated to determine its mass accumulation rate (MAR) and grain size in post-middle Oligocene sediments from DSDP Sites 597 to 602 , which lie along $19^{\circ} \mathrm{S}$ on the East Pacific Rise. The rate of eolian deposition has been very low (about 2 to $3 \mathrm{mg} /\left(\mathrm{cm}^{2} \times 10^{3}\right.$ yr.) and reasonably consistent at all the sites, except that the MAR of the eolian component at Site 597 increases to an average of about $8 \mathrm{mg} /\left(\mathrm{cm}^{2} \times 10^{3} \mathrm{yr}\right.$.) between 17 and $19 \mathrm{Ma}$. Eolian grain size at Site 598 changes dramatically at $10.5 \mathrm{Ma}$ from $7.2 \phi$ in younger sediments to $8.2 \phi$ in older material and may provide evidence of an intensification of zonal winds that took place in response to Antarctic ice formation just prior to that time. Pliocene to Pleistocene samples from Leg 92 show no evidence of the severalfold increase in dust accumulation and grain size recorded at Northern Hemisphere locations at the time corresponding to the onset of Northern Hemisphere glaciation. The low and constant flux of eolian material indicates that South America was consistently vegetated from post-middle Oligocene time to the present.
\end{abstract}

\section{INTRODUCTION}

\section{Previous Estimates of Past Atmospheric Circulation}

DSDP Leg 92 was conducted to investigate the hydrogeology of the East Pacific Rise along a transect at $19^{\circ} \mathrm{S}$. The Glomar Challenger drilled 19 holes at six sites (Fig. 1), recovering sediments that are a mixture of nannofossil ooze and clay. The sediments contain little terrigenous material (site chapters, this volume), as indicated by their low quartz content. This terrigenous component, which far from land is of eolian origin, was analyzed to determine first-order Oligocene to Pleistocene paleoenvironmental and paleoclimatic variations in the Southern Hemisphere's trade wind zone.

Knowledge of the intensity of atmospheric circulation and its variation through geologic time has long been recognized as important to an understanding of past climatic variation. However, estimates of wind intensity during the geologic past are rare. Several indirect methods for estimating past atmospheric circulation patterns have been presented that depend upon the correlation between atmospheric and oceanic circulation patterns. For example, relative wind intensities have been inferred from studies of past biological productivity as indicated by the fossil plankton assemblages under wind-driven oceanic circulation systems (Ingle, 1973; van Andel et al., 1975) and the distribution of temperature-sensitive marine micro-organisms (Climap Project Members, 1976, 1981; Moore et al., 1980). Some computer models of atmospheric circulation patterns based on indirect climatological information about periods of glaciation also have wind intensity among their outputs (Gates, 1976; Manabe and Hahn, 1977).

\footnotetext{
${ }^{1}$ Leinen, M., Rea, D. K., et al., Init. Repts. DSDP, 92: Washington (U.S. Govt. Printing Office).

2 Address: Oceanography Program, Department of Atmospheric and Oceanic Science, The University of Michigan, Ann Arbor, MI 48109.
}

\section{Eolian Sedimentation}

Oceanographers have known for a long time that dust is transported to the oceans from the continents (Maury, 1855; Radczewski, 1939; Rex and Goldberg, 1958; Prospero and Bonatti, 1969; Windom, 1975; Windom and Chamberlain, 1978). These fine grains of quartz and clay comprise an important component of the sediment recovered from the central portions of ocean basins (Windom, 1969; Ferguson et al., 1970).

The eolian material provides a record of the intensity of atmospheric circulation and climatic conditions in the continental source area (Rea and Janecek, 1982; Janecek and Rea, 1983). Eolian material is transported to the ocean basins in greater quantities during times of global aridity, when the sparseness of vegetation allows greater amounts of material to be entrained into the atmosphere. Volcanoes can also contribute ash to the wind-borne sediments, and at times the material from these episodic sources can be voluminous.

Another factor controlling eolian deposition is the strength of the global winds. The strength of these winds depends in the most simple sense upon the pole-to-equator temperature gradient (Lamb and Woodroffe, 1970); the larger the gradient, the greater the velocity of zonal winds. During periods of polar cooling and glacial advance, this temperature gradient steepens, and wind intensity increases (Climap Project Members, 1976, 1981; Gates, 1976). Conversely, during periods of global warming, atmospheric circulation would be expected to decrease in intensity.

The grain size of eolian material initially reflects that of the parent soil, with winds carrying grains as coarse as medium-grained sand (Gillette, 1981). The larger grains begin to settle out immediately, until over time and distance wind-borne eolian material is characterized by grains 1 to $10 \mu \mathrm{m}$ in diameter (Gillette, 1974; Gillette and Walker, 1977). This material may be carried around the world 


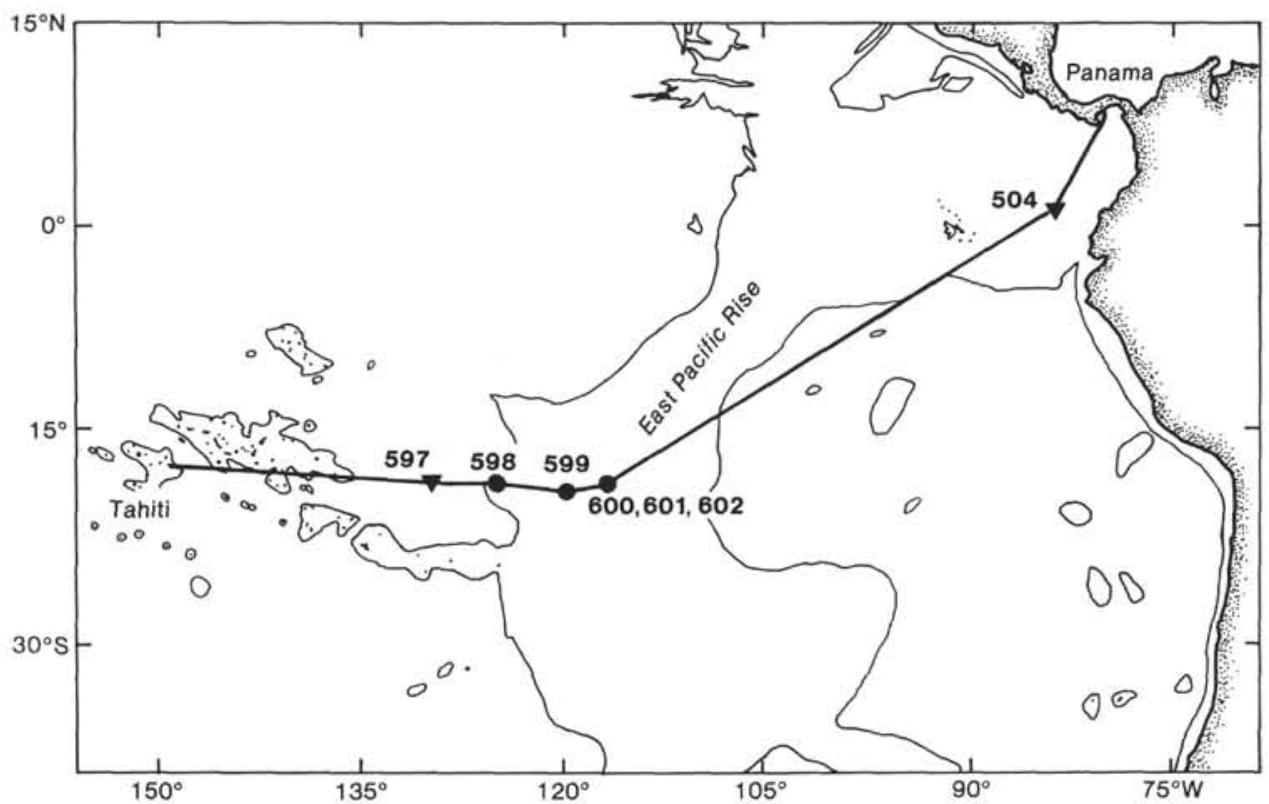

Figure 1. Location of Leg 92 drill sites in South Pacific Ocean. Inverted triangles denote reentry sites.

and is removed from the atmosphere by being rained out (Jackson et al., 1973).

Only a few downcore studies of eolian material have been made. Parkin and co-workers (Parkin, 1974; Parkin and Shackleton, 1973; Parkin and Padgham, 1975) studied two cores from the continental margin of Africa and found that the abundance and size of quartz grains larger than $7 \mu \mathrm{m}$ increased during glacial periods and inferred stronger trade winds. Stronger trade winds during glacial periods were also suggested by Sarnthein et al. (1982), who studied sediments from the northwest continental margin of Africa. Theide (1979) has shown that the concentration of quartz in the sediment east of Australia and New Zealand was greater during the last glacial maximum than it is now.

Several investigators have studied long-term (millions of years) variations in eolian accumulation rates. Rea and Janecek (1981a, b) examined Late Cretaceous eolian deposition at DSDP Site 463 in the mid-Pacific mountains. They found that when temperature gradients remained constant, changes in eolian accumulation rates generally coincided with known changes in sea level; the coincidence suggests that source area supply was the controlling factor during this period. Leinen and Heath (1981) analyzed the quartz component, and Janecek and Rea (1983) analyzed the total eolian component, in the sediment from North Pacific Core LL44-GPC-3. These studies also suggested that eolian accumulation rates are related to the climate of the source area, with a low accumulation rate during the Paleogene reflecting the temperate and more humid environment of the earlier Cenozoic and a higher accumulation rate during the $\mathrm{Ne}$ ogene recording increasing aridity. Rea and Janecek (1982) studied the deposition of eolian material from the late Miocene to the Pleistocene as recorded in several North Pacific DSDP cores. Their results indicate that at the onset of Northern Hemisphere glaciation, zonal winds increased in intensity by approximately $50 \%$ and that at the same time the flux of dust to the deep sea increased fivefold. Other studies have shown that the grain size of the eolian material increased significantly during the Oligocene, Miocene, and mid-Pliocene, a reflection of the increased wind intensity resulting from polar isolation and ice buildup during those periods (Rea et al., 1985).

\section{METHODS OF ANALYSIS}

The data we generate are the mass accumulation rate (MAR) of the total eolian component (not just the quartz component, which may comprise only 20 to $30 \%$ of the wind-transported sediment; Glaccum and Prospero, 1980) and the median grain size of the eolian component. We assume that variations in the grain size of the eolian component and variations in the mass accumulation rate reflect fluctuations in the paleoenvironment and paleoclimate of the trade wind zones in the South Pacific.

The rate of deposition can be determined in two ways. The first method is to divide a measured length of section by the time required for it to accumulate. This method gives the length deposited per unit time, a result referred to as the linear sedimentation rate (LSR). However, compaction causes a reduction in porosity downsection because of the weight of the overlying sediments. This reduction can be as great as 30 or $50 \%$ in the lowermost sections of longer cores. Also, because the LSR measures only the thickness of the sediment deposited, it cannot be used to determine fluxes of material to the seafloor.

The second method is to measure the dry mass flux of material across the sediment/water interface and has a result referred to as the mass accumulation rate. Unlike the LSR, which measures only the thickness of the sediment deposited per unit tine, the MAR is an expression of the mass of sedimentary material accumulating per unit area and time. The MAR is not affected by variations in porosity and allows samples from different places and depths to be compared. The MAR is the product of the LSR and dry bulk density and is a quantitative measure of the rate at which material is added to the sediment. The MAR of the eolian component was determined by finding the product of the weight percent non-authigenic, inorganic, crystalline component and the total MAR of each sample (Table 1).

The mineral component which, far from land, represents eolian material (Leinen and Heath, 1981) was isolated by a series of extractions to remove $\mathrm{CaCO}_{3}$, opaline silica, $\mathrm{Fe} / \mathrm{Mn}$ oxides and hydroxides, and zeolites. Details of this procedure are given in Rea and Janecek (1981b).

The grain size of the eolian component was measured for the 6 to $10 \phi$ size fraction at $0.33 \phi$ intervals by using a Coulter Counter model 
Table 1. Data for calculation of mass accumulation rates and grain size of the eolian component of sediments from Sites 597, 598, 599, 600,601 , and 602 .

\begin{tabular}{|c|c|c|c|c|c|c|c|c|c|}
\hline $\begin{array}{c}\text { Sample } \\
\text { (interval in } \mathrm{cm} \text { ) }\end{array}$ & $\begin{array}{l}\text { Sub-bottom } \\
\text { depth } \\
\text { (m) }\end{array}$ & $\begin{array}{c}\text { Age } \\
(\mathrm{m} . \mathrm{y} .)\end{array}$ & $\begin{array}{l}\text { Porosity } \\
(\%)\end{array}$ & $\begin{array}{l}\text { Dry bulk } \\
\text { density } \\
\left(\mathrm{g} / \mathrm{cm}^{2}\right)\end{array}$ & $\begin{array}{c}\text { LSR } \\
(\mathrm{m} / \mathrm{m} \cdot \mathrm{y} .)\end{array}$ & $\begin{array}{c}\text { MAR } \\
\left(\mathrm{mg} /\left[\mathrm{cm}^{2} \times 10^{3} \mathrm{yr} .\right]\right)\end{array}$ & $\begin{array}{l}\text { Eolian } \\
\text { fraction } \\
\text { (wt. \%) }\end{array}$ & $\begin{array}{c}\text { Eolian } \\
\text { MAR } \\
\left(\mathrm{mg} /\left[\mathrm{cm}^{2} \times 10^{3} \text { yr. }\right]\right)\end{array}$ & $\begin{array}{l}\text { Median } \\
\text { eolian } \\
\text { grain size } \mathrm{a}^{\mathrm{a}} \\
(\phi)\end{array}$ \\
\hline \multicolumn{10}{|c|}{ Hole 597} \\
\hline $1-1,14-18$ & 0.16 & 1.0 & 81.65 & 0.486 & 0.11 & 5.3 & 19.28 & 1.0 & 6.93 \\
\hline $1-1,96-100$ & 0.98 & 9.0 & 82.03 & 0.476 & 0.11 & 5.2 & 8.65 & 0.5 & 9.83 \\
\hline $1-2,46-50$ & 1.98 & 14.7 & 68.42 & 0.837 & 0.93 & 77.8 & 2.55 & 2.0 & 8.38 \\
\hline $1-3,6-10$ & 3.08 & 15.9 & 69.54 & 0.807 & 0.93 & 75.1 & 3.34 & 2.5 & 8.32 \\
\hline $1-3,96-100$ & 3.98 & 16.9 & 66.83 & 0.879 & 0.93 & 81.7 & 2.03 & 1.7 & 9.02 \\
\hline $2-1,6-10$ & 4.68 & 17.1 & 68.00 & 0.848 & 6.71 & 569.0 & 2.72 & 15.5 & 9.63 \\
\hline $2-1,96-100$ & 5.58 & 17.2 & 67.21 & 0.869 & 6.71 & 583.0 & 1.79 & 10.4 & 9.61 \\
\hline $2-2,46-50$ & 6.58 & 17.4 & 65.59 & 0.912 & 6.71 & 611.9 & 1.41 & 8.6 & 7.96 \\
\hline $2-3,5-9$ & 7.67 & 17.5 & 62.08 & 1.009 & 6.71 & 676.9 & 0.89 & 6.0 & 7.69 \\
\hline $2-3,96-100$ & 8.58 & 17.7 & 60.24 & 1.054 & 6.71 & 707.0 & 0.81 & 5.7 & 7.51 \\
\hline $2-4,46-50$ & 9.58 & 17.8 & 61.25 & 1.027 & 6.71 & 689.0 & 0.77 & 5.3 & 8.70 \\
\hline $2-5,6-10$ & 10.68 & 18.0 & 61.89 & 1.010 & 6.71 & 677.4 & 0.89 & 6.0 & 7.63 \\
\hline $2-5,96-100$ & 11.58 & 18.1 & 60.94 & 1.035 & 6.71 & 694.5 & 1.55 & 10.8 & 7.36 \\
\hline $2-6,46-50$ & 12.58 & 18.3 & 51.18 & 1.294 & 6.71 & 868.1 & 2.71 & 23.5 & 7.58 \\
\hline $2-7,6-10$ & 13.68 & 18.4 & 50.70 & 1.307 & 6.71 & 695.5 & 0.84 & 5.8 & 8.69 \\
\hline $3-1,6-10$ & 14.28 & 18.5 & 58.90 & 1.089 & 6.71 & 730.9 & 2.02 & 14.8 & $7.51^{b}$ \\
\hline $3-1,96-100$ & 15.18 & 18.7 & 59.02 & 1.086 & 6.71 & 728.6 & 0.73 & 5.3 & 7.33 \\
\hline $3-2,46-50$ & 16.18 & 19.3 & 58.85 & 1.091 & 1.12 & 122.1 & 0.91 & 1.1 & 8.16 \\
\hline $3-3,8-12$ & 17.30 & 20.3 & 59.06 & 1.085 & 1.12 & 121.5 & 1.25 & 1.5 & 8.40 \\
\hline $3-3,96-100$ & 18.18 & 21.1 & 58.78 & 1.092 & 1.12 & 122.3 & 1.14 & 1.4 & 8.28 \\
\hline $3-4,46-50$ & 19.18 & 22.0 & 59.52 & 1.073 & 1.12 & 120.1 & 1.17 & 1.4 & 7.29 \\
\hline $3-5,6-10$ & 20.28 & 22.4 & 57.06 & 1.138 & 3.07 & 349.3 & 1.16 & 4.1 & 8.32 \\
\hline $3-5,96-100$ & 21.18 & 22.6 & 58.07 & 1.111 & 3.07 & 341.1 & 0.88 & 3.0 & 7.87 \\
\hline $3-6,46-50$ & 22.18 & 23.0 & 59.41 & 1.076 & 3.07 & 330.2 & 0.73 & 2.4 & 7.41 \\
\hline $4-1,6-10$ & 23.88 & 23.5 & 59.90 & 1.063 & 3.07 & 326.2 & 0.41 & 1.3 & $8.27^{b}$ \\
\hline $4-1,96-100$ & 24.78 & 23.8 & 60.93 & 1.035 & 3.07 & 317.8 & 0.50 & 1.6 & $8.44^{b}$ \\
\hline $4-2,46-50$ & 25.78 & 24.1 & 61.81 & 1.012 & 3.07 & 310.7 & 0.47 & 1.5 & 8.57 \\
\hline $4-3,6-10$ & 26.88 & 24.5 & 62.61 & 0.991 & 3.07 & 304.2 & 0.47 & 1.4 & 8.20 \\
\hline $4-3,96-100$ & 27.78 & 24.8 & 61.37 & 1.024 & 3.07 & 314.3 & 0.42 & & 8.26 \\
\hline $4-4,46-50$ & 28.78 & 24.9 & 59.78 & 1.066 & 7.69 & 819.6 & 0.58 & 4.8 & 8.27 \\
\hline $4-5,6-10$ & 29.88 & 25.1 & 60.17 & 1.056 & 7.69 & 811.7 & 0.40 & 3.2 & 8.41 \\
\hline $4-5,96-100$ & 30.78 & 25.2 & 60.87 & 1.037 & 7.69 & 797.4 & 0.33 & 2.6 & 8.14 \\
\hline $4-6,46-50$ & 31.78 & 25.3 & 60.47 & 1.048 & 7.69 & 805.5 & 0.33 & 2.7 & $8.46^{b}$ \\
\hline $6-1,6-10$ & 43.08 & 26.8 & 62.31 & 0.999 & 7.69 & 768.1 & 3.26 & 25.0 & 7.36 \\
\hline $6-1,96-100$ & 43.98 & 26.9 & 66.75 & 0.881 & 7.69 & 677.6 & 2.36 & 16.0 & 7.44 \\
\hline $6-2,46-50$ & 44.98 & 27.0 & & & 7.69 & & 2.06 & & $7.85^{b}$ \\
\hline $6-3,6-10$ & 46.08 & 27.2 & 64.12 & 0.951 & 7.69 & 731.2 & 29.38 & 726.7 & 8.27 \\
\hline $6-3,96-100$ & 46.98 & 27.3 & 54.18 & 1.214 & 7.69 & 933.7 & 4.98 & 46.5 & 7.44 \\
\hline $6-4,46-50$ & 47.98 & 27.4 & 54.10 & 1.216 & 7.69 & 935.3 & 3.40 & 31.8 & $7.57^{b}$ \\
\hline $6-5,96-100$ & 49.98 & 27.7 & 56.88 & 1.143 & 7.69 & 878.7 & 1.71 & 15.0 & 8.09 \\
\hline $6-6,46-50$ & 50.98 & 27.8 & 57.06 & 1.138 & 7.69 & 875.0 & 2.66 & 23.3 & 7.40 \\
\hline \multicolumn{10}{|c|}{ Hole 598} \\
\hline $1-1,11-14$ & 0.13 & 0.2 & 70.73 & 0.776 & 0.53 & 41.1 & 1.55 & 0.6 & 7.88 \\
\hline $1-1,101-104$ & 1.03 & 1.9 & 68.07 & 0.846 & 1.05 & 88.8 & 4.04 & 3.6 & $7.19^{b}$ \\
\hline $1-2,51-54$ & 2.03 & 2.9 & 68.14 & 0.844 & 1.05 & 88.7 & 3.50 & 3.1 & 6.96 \\
\hline $1-3,8-11$ & 3.10 & 3.9 & 62.14 & 1.003 & 1.05 & 105.3 & 0.85 & 0.9 & 7.02 \\
\hline $1-3,101-104$ & 4.03 & 5.0 & 63.15 & 0.977 & 1.05 & 102.5 & 1.05 & 1.1 & 7.22 \\
\hline $1-4,51-54$ & 5.03 & 5.7 & 65.58 & 0.912 & 1.31 & 119.5 & 2.91 & 3.5 & 7.06 \\
\hline $2-1,11-14$ & 6.33 & 6.6 & 62.74 & 0.987 & 1.31 & 129.3 & 0.30 & 0.4 & 6.88 \\
\hline $2-1,101-104$ & 7.23 & 7.1 & 61.45 & 1.022 & 1.86 & 190.0 & 0.54 & 1.0 & $7.26^{\mathrm{b}}$ \\
\hline $2-2,51-54$ & 8.23 & 7.7 & 62.77 & 0.998 & 1.86 & 185.7 & 4.06 & 7.5 & 6.94 \\
\hline $2-3,11-14$ & 9.33 & 8.2 & 64.15 & 0.950 & 3.09 & 293.6 & 6.49 & 19.1 & 7.39 \\
\hline $2-3,101-104$ & 10.23 & 8.5 & 61.81 & 1.012 & 3.09 & 312.7 & 0.92 & 2.9 & 6.98 \\
\hline $2-4,51-54$ & 11.23 & 8.8 & 63.01 & 0.980 & 3.09 & 302.9 & 1.24 & 3.8 & 7.18 \\
\hline $2-5,11-14$ & 12.33 & 9.2 & 64.37 & 0.944 & 3.09 & 291.8 & 0.93 & 2.7 & 7.21 \\
\hline $2-5,101-104$ & 13.23 & 9.5 & 63.85 & 0.958 & 2.40 & 229.9 & 1.45 & 3.3 & 7.07 \\
\hline $2-6,51-54$ & 14.23 & 10.0 & 61.19 & 1.029 & 2.40 & 246.8 & 0.86 & 2.1 & 8.64 \\
\hline $2-7,10-14$ & 15.33 & 10.4 & 65.77 & 0.907 & 2.40 & 217.7 & 1.16 & 2.5 & 7.45 \\
\hline $3-1,11-14$ & 15.93 & 10.7 & 67.01 & 0.874 & 2.40 & 209.8 & 1.98 & 4.2 & 7.92 \\
\hline $3-1,101-104$ & 16.83 & 11.8 & 66.09 & 0.899 & 0.77 & 69.2 & 1.09 & 0.8 & 8.69 \\
\hline $3-2,51-54$ & 17.83 & 12.1 & 61.70 & 1.015 & 5.77 & 585.6 & 0.80 & 4.7 & 7.23 \\
\hline $3-3,11-14$ & 18.93 & 12.3 & 64.19 & 0.949 & 5.77 & 547.6 & 0.55 & 3.0 & .7 .76 \\
\hline $3-3,101-104$ & 19.83 & 12.5 & 66.65 & 0.884 & 5.77 & 510.0 & 0.60 & 3.1 & 8.26 \\
\hline $3-4,51-54$ & 20.83 & 12.7 & 63.60 & 0.965 & 5.77 & 556.6 & 0.54 & 3.0 & 8.59 \\
\hline $3-5,11-14$ & 21.93 & 12.9 & 64.68 & 0.936 & 5.77 & 540.1 & 0.61 & 3.3 & 8.49 \\
\hline $3-5,101-104$ & 22.83 & 13.0 & 65.19 & 0.923 & 5.77 & 532.3 & 0.67 & 3.6 & 7.95 \\
\hline $3-6,51-54$ & 23.83 & 13.1 & 67.64 & 0.857 & 5.77 & 494.7 & 0.52 & 2.6 & 8.29 \\
\hline $3-C C, 12-15$ & 24.69 & 13.4 & 64.62 & 0.938 & 5.77 & 541.0 & 0.31 & 1.7 & 8.90 \\
\hline $4-1,11-14$ & 25.53 & 13.5 & 67.57 & 0.859 & 5.77 & 495.9 & 0.61 & 3.0 & 8.44 \\
\hline $4-1,101-104$ & 26.43 & 13.6 & 69.20 & 0.816 & 5.77 & 470.9 & 0.79 & 3.7 & 8.39 \\
\hline
\end{tabular}


Table 1 (continued).

\begin{tabular}{|c|c|c|c|c|c|c|c|c|c|}
\hline $\begin{array}{c}\text { Sample } \\
\text { (interval in cm) }\end{array}$ & $\begin{array}{l}\text { Sub-bottom } \\
\text { depth } \\
\text { (m) }\end{array}$ & $\begin{array}{l}\text { Age } \\
\text { (m.y.) }\end{array}$ & $\begin{array}{l}\text { Porosity } \\
(\%)\end{array}$ & $\begin{array}{l}\text { Dry bulk } \\
\text { density } \\
\left(\mathrm{g} / \mathrm{cm}^{2}\right)\end{array}$ & $\underset{(\mathrm{m} / \mathrm{m} . \mathrm{y} .)}{\mathrm{LSR}}$ & $\underset{\left(\mathrm{mg} /\left[\mathrm{cm}^{2} \times 10^{3} \mathrm{yr} \cdot\right]\right)}{\operatorname{MAR}}$ & $\begin{array}{l}\text { Eolian } \\
\text { fraction } \\
\text { (wt.\%) }\end{array}$ & $\begin{array}{c}\text { Eolian } \\
\text { MAR } \\
\left(\mathrm{mg} /\left[\mathrm{cm}^{2} \times 10^{3} \mathrm{yr} .\right]\right)\end{array}$ & $\begin{array}{c}\text { Median } \\
\text { eolian } \\
\text { grain size } \\
\qquad(\phi)\end{array}$ \\
\hline \multicolumn{10}{|l|}{ Hole 598 (Cont.) } \\
\hline $4-2,51-54$ & 27.43 & 13.8 & 67.33 & 0.866 & 5.77 & 499.6 & & & 8.14 \\
\hline $4-3,11-14$ & 28.53 & 14.0 & 64.44 & 0.942 & 5.77 & 543.7 & 0.78 & 4.2 & 8.58 \\
\hline $4-3,101-104$ & 29.43 & 14.2 & 68.89 & 0.824 & 5.77 & 475.7 & 0.70 & 3.3 & 7.94 \\
\hline $4-4,51-54$ & 30.43 & 14.3 & 62.58 & 0.992 & 8.11 & 804.2 & 0.37 & 3.0 & 8.08 \\
\hline $4-5,11-14$ & 31.53 & 14.4 & 62.48 & 0.994 & 8.11 & 806.4 & 0.33 & 2.7 & 8.54 \\
\hline $4-5,101-104$ & 32.53 & 14.5 & 66.59 & 0.885 & 8.11 & 718.0 & 0.38 & 2.7 & 8.27 \\
\hline $4-6,35-38$ & 33.27 & 14.6 & 65.77 & 0.907 & 8.11 & 735.7 & 0.65 & 4.8 & 8.30 \\
\hline $5-1,11-14$ & 35.13 & 14.9 & 68.46 & 0.836 & 8.11 & 677.8 & 0.38 & 2.6 & 9.01 \\
\hline $5-1,101-104$ & 36.03 & 15.0 & 68.95 & 0.823 & 8.11 & 667.3 & 0.28 & 1.9 & 9.17 \\
\hline $5-2,51-54$ & 27.03 & 15.1 & 68.64 & 0.831 & 8.11 & 673.9 & 0.95 & 6.4 & 8.65 \\
\hline $5-3,11-14$ & 38.13 & 15.2 & 68.61 & 0.832 & 8.11 & 674.6 & 0.50 & 3.4 & 8.06 \\
\hline $5-3,101-104$ & 39.03 & 15.4 & 70.84 & 0.773 & 8.11 & 626.7 & 0.97 & 6.1 & 8.32 \\
\hline $5-4,51-54$ & 40.03 & 15.5 & 68.68 & 0.830 & 8.11 & 673.0 & 0.50 & 3.4 & 7.48 \\
\hline $5-5,11-14$ & 41.13 & 15.6 & 67.53 & 0.861 & 8.11 & 697.9 & 0.56 & 3.9 & $8.36^{\mathrm{b}}$ \\
\hline $5-5,101-104$ & 42.03 & 15.7 & 67.34 & 0.866 & 8.11 & 701.9 & 0.73 & 5.1 & $7.26^{\mathrm{b}}$ \\
\hline $5-6,51-54$ & 43.03 & 15.8 & 64.01 & 0.954 & 8.11 & 773.5 & 1.20 & 9.3 & 7.69 \\
\hline \multicolumn{10}{|c|}{ Hole 599} \\
\hline $1-1,11-14$ & 0.13 & 0.25 & 59.03 & 1.086 & 0.53 & 57.5 & 0.72 & 0.4 & 6.77 \\
\hline $1-1,101-104$ & 1.03 & 1.90 & 63.54 & 0.966 & 0.99 & 95.7 & 2.15 & 2.1 & $7.02^{\mathrm{b}}$ \\
\hline $1-2,51-54$ & 2.03 & 2.90 & 59.88 & 1.063 & 0.99 & 105.3 & 1.47 & 1.6 & $6.94^{b}$ \\
\hline $1-3,11-14$ & 3.13 & 3.80 & 56.60 & 1.150 & 5.35 & 615.3 & 0.58 & 3.6 & 7.18 \\
\hline $1-3,101-104$ & 4.30 & 4.00 & 56.55 & 1.150 & 5.35 & 615.3 & 0.58 & 3.6 & 7.43 \\
\hline $1-4,51-54$ & 5.13 & 4.10 & 60.07 & 1.058 & 5.35 & 566.1 & 0.66 & 3.7 & $8.77^{\mathrm{b}}$ \\
\hline $1-5,11-14$ & 6.13 & 4.30 & 58.71 & 1.094 & 5.35 & 585.4 & 0.21 & 1.2 & 8.22 \\
\hline $1-5,101-104$ & 7.03 & 4.50 & 59.42 & 1.075 & 5.35 & 575.3 & 0.34 & 2.0 & 8.52 \\
\hline 1-CC, 4-7 & 7.76 & 4.60 & 55.88 & 1.169 & 5.35 & 1700.0 & 0.38 & 6.5 & 8.49 \\
\hline $2-1,11-14$ & 8.03 & 4.70 & 61.73 & 1.014 & 5.35 & 542.6 & 0.43 & 2.3 & 6.65 \\
\hline $2-1,101-104$ & 8.93 & 4.80 & 59.75 & 1.067 & 5.35 & 570.6 & 0.18 & 1.0 & 7.84 \\
\hline $2-2,41-44$ & 9.83 & 5.00 & 67.85 & 0.852 & 5.35 & 455.8 & 0.36 & 1.6 & 7.12 \\
\hline $2-3,11-14$ & 11.03 & 5.20 & 69.09 & 0.819 & 5.35 & 438.1 & 0.53 & 2.3 & 6.28 \\
\hline $2-3,101-104$ & 11.93 & 5.40 & 56.38 & 1.559 & 14.54 & 2267.2 & 6.19 & 140.3 & 7.75 \\
\hline $2-4,51-54$ & 12.93 & 5.50 & 71.59 & 0.753 & 14.54 & 1094.7 & 0.12 & 1.3 & 6.51 \\
\hline $2-5,11-14$ & 14.03 & 5.50 & 62.71 & 0.988 & 14.54 & 1436.8 & 0.15 & 2.2 & 6.54 \\
\hline $2-5,101-104$ & 14.93 & 5.60 & 74.05 & 0.688 & 14.54 & 999.9 & 0.31 & 3.1 & 6.58 \\
\hline $2-6,51-54$ & 15.93 & 5.70 & 70.84 & 0.773 & 14.54 & 1123.5 & 0.52 & 5.8 & 7.46 \\
\hline $2-7,11-14$ & 17.03 & 5.80 & 76.88 & 0.613 & 14.54 & 890.9 & 1.06 & 9.4 & $8.64^{\mathrm{b}}$ \\
\hline $3-1,11-14$ & 17.63 & 5.80 & 69.95 & 0.810 & 14.54 & 1177.0 & 0.54 & 6.4 & 6.74 \\
\hline $3-1,101-104$ & 18.53 & 5.90 & 70.11 & 0.792 & 14.54 & 1151.6 & 0.18 & 2.1 & 6.25 \\
\hline $3-2,51-54$ & 19.53 & 5.90 & 67.95 & 0.849 & 14.54 & 1234.7 & 0.41 & 5.1 & 6.85 \\
\hline $3-3,11-14$ & 20.63 & 6.00 & 66.92 & 0.876 & 14.54 & 1274.1 & 0.32 & 4.1 & 6.89 \\
\hline $3-3,101-104$ & 21.80 & 6.10 & 62.58 & 0.992 & 14.54 & 1441.8 & 0.37 & 5.3 & 7.14 \\
\hline $3-4,51-54$ & 22.52 & 6.10 & 69.69 & 0.803 & 14.54 & 1167.7 & 0.75 & 8.8 & 6.63 \\
\hline $3-5,11-14$ & 23.63 & 6.20 & 68.12 & 0.845 & 14.54 & 1228.5 & 1.07 & 13.1 & $8.23^{\mathrm{b}}$ \\
\hline $3-5,101-104$ & 24.53 & 6.30 & 70.12 & 0.792 & 14.54 & 1151.3 & 23.20 & 267.1 & 7.26 \\
\hline $3-6,51-54$ & 25.52 & 6.30 & 69.36 & 0.812 & 14.54 & 1180.5 & 3.09 & 36.5 & 6.96 \\
\hline $4-1,11-14$ & 27.23 & 6.50 & 59.48 & 1.074 & 14.54 & 1561.3 & 0.79 & 12.3 & 7.54 \\
\hline $4-1,101-104$ & 28.13 & 6.50 & 70.82 & 0.773 & 14.54 & 1124.2 & 2.91 & 32.7 & 6.37 \\
\hline $4-2,51-54$ & 29.13 & 6.60 & 69.99 & 0.795 & 14.54 & 1156.1 & 1.51 & 17.5 & 7.54 \\
\hline $4-3,11-14$ & 30.23 & 6.70 & 65.32 & 0.919 & 14.54 & 1136.1 & 7.69 & 90.4 & 7.78 \\
\hline $4-3,101-104$ & 31.13 & 6.80 & & & 2.86 & & 0.65 & & 7.33 \\
\hline $4-4,51-54$ & 32.13 & 7.20 & 68.54 & 0.834 & 2.86 & 238.4 & 1.18 & 2.8 & 7.29 \\
\hline $4-5,11-14$ & 33.23 & 7.50 & 68.83 & 0.826 & 2.86 & 236.2 & 0.15 & 0.4 & $6.82^{b}$ \\
\hline $4-5,101-104$ & 34.40 & 8.00 & 66.57 & 0.886 & 2.86 & 253.4 & 0.49 & 1.2 & 7.63 \\
\hline $4-6,51-54$ & 35.13 & 8.10 & 70.18 & 0.790 & 12.00 & 948.1 & 0.79 & 7.5 & 7.58 \\
\hline \multicolumn{10}{|c|}{ Hole 599B } \\
\hline $2-1,11-14$ & 32.33 & 8.2 & 65.73 & 0.908 & 12.00 & 1089.8 & 0.72 & 7.9 & $6.88^{\mathrm{b}}$ \\
\hline $2-1,101-104$ & 33.23 & 8.3 & 67.51 & 0.861 & 12.00 & 1033.1 & 0.71 & 7.3 & 6.54 \\
\hline $2-2,51-54$ & 34.23 & 8.4 & 70.05 & 0.794 & 12.00 & 952.4 & 1.15 & 11.0 & $7.06^{\mathrm{b}}$ \\
\hline $2-3,11-14$ & 35.33 & 8.4 & 60.90 & 1.036 & 12.00 & 1243.6 & 0.23 & 2.9 & $7.94^{\mathrm{b}}$ \\
\hline $2-3,101-104$ & 36.23 & 8.5 & 72.14 & 0.738 & 12.00 & 885.9 & 0.44 & 3.9 & 6.78 \\
\hline $2-4,51-54$ & 37.23 & 8.6 & 62.13 & 1.004 & 12.00 & 1204.3 & 0.98 & 11.8 & 7.05 \\
\hline $2-5,11-14$ & 38.33 & 8.7 & 71.44 & 0.757 & 12.00 & 908.2 & 1.84 & 16.7 & 8.70 \\
\hline $2-5,101-104$ & 39.23 & 8.7 & 65.50 & 0.914 & 12.00 & 1097.2 & 3.19 & 35.0 & 8.15 \\
\hline $2-6,51-54$ & 40.23 & 8.8 & 69.90 & 0.798 & 12.00 & 957.2 & 5.50 & 52.7 & 6.58 \\
\hline $2-7,11-14$ & 41.33 & 8.9 & 69.69 & 0.803 & 12.00 & 963.8 & 4.00 & 38.6 & \\
\hline \multicolumn{10}{|c|}{ Hole 600} \\
\hline $1-1,11-14$ & 0.13 & & 71.93 & 0.744 & & & 0.35 & & $7.83^{\mathrm{b}}$ \\
\hline $1-1,101-104$ & 1.03 & & 58.50 & 1.100 & & & 0.24 & & $8.29^{b}$ \\
\hline
\end{tabular}


Table 1 (continued).

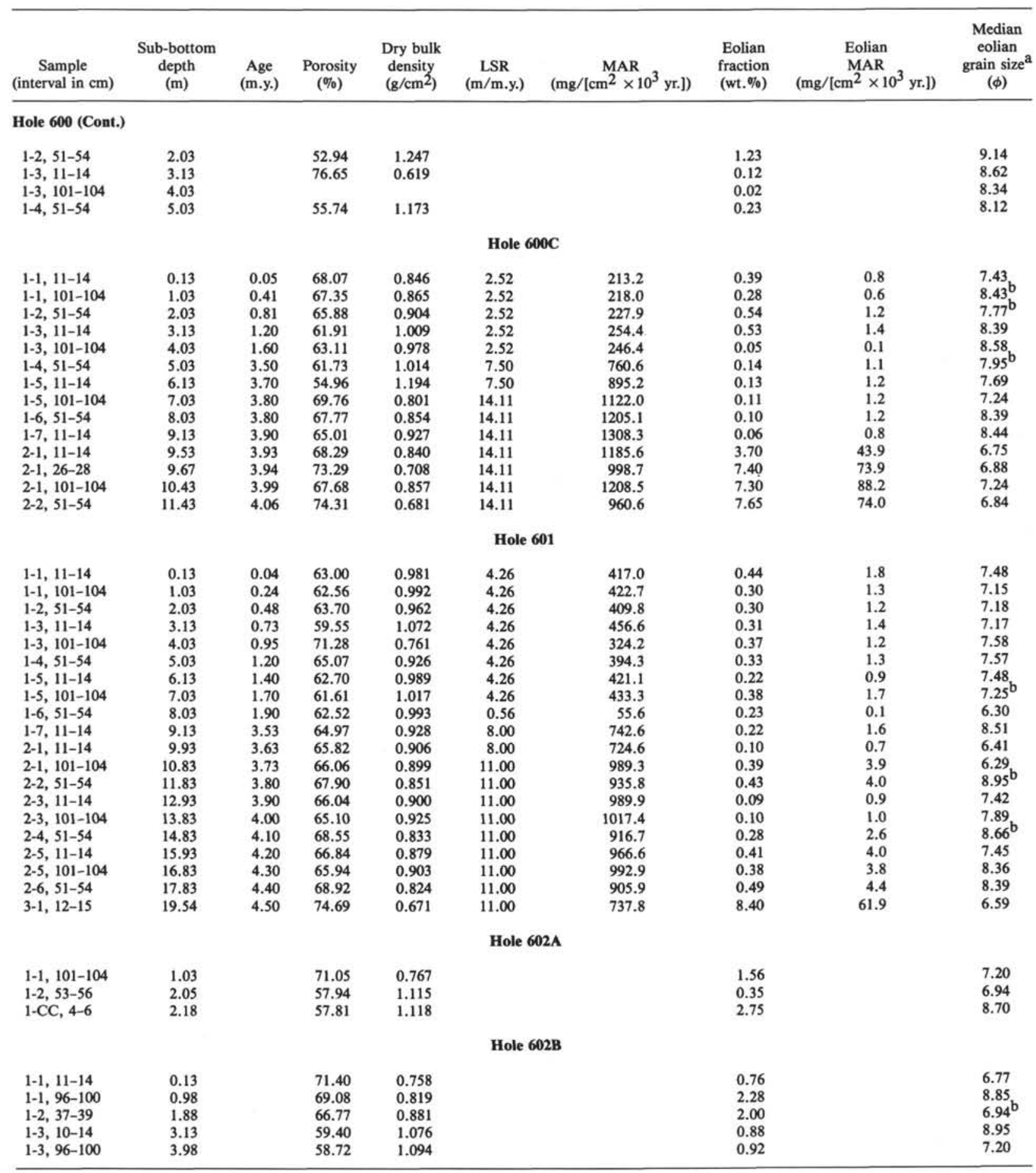

a $\phi_{50}$ of Folk (1974).

Samples for which two data sets had to be averaged to determine eolian grain size (see Methods of Analysis).

TA-II particle size analyzer. ${ }^{3}$ The median grain size values $\left(\phi_{50}\right.$ of Folk, 1974) were determined by normalizing the primary data to $100 \%$ and averaging duplicate runs for each sample interval; the resulting precision was \pm 0.05 (Table 1). Several samples gave widely divergent values, and to improve the precision of the size found for these samples a second set of duplicate runs was completed and averaged with the first.

\footnotetext{
${ }^{3}$ The 6 to $10 \phi$ size fraction is equivalent to the 16 to $1 \mu \mathrm{m}$ size fraction. $\phi=-\log _{2} D$
} where $D$ is grain diameter in millimeters.
The accuracy of the mass accumulation rate data for the eolian component is difficult to determine and depends partly on the accuracy of the biostratigraphy and partly upon the accuracy of laboratory procedure. Inaccuracies in the placement of the biostratigraphic boundaries (which for this study were taken from the various site chapters; this volume), deviations from the constant LSR that was assumed between biostratigraphic boundaries, and inaccuracies in the assignment of absolute ages to the biostratigraphic boundaries combine to produce errors larger than a few percent. Furthermore, the remarkably low amount of eolian material present $(0.5 \%$ as compared 
with 5 to $50 \%$ in Northern Hemisphere sediments) and the presence of some volcanic glass in most samples prevent us from attaching geological significance to any except the largest and most obvious changes.

We sampled the Leg 92 cores at approximately 1-m intervals (Table 1). Since sedimentation rates vary by 2 orders of magnitude, the spacing of the resulting data for eolian MAR and grain size varies widely when plotted versus sample age (Figs. 2 to 5). The apparent smoothing of the data from the younger, upper portions of the holes should be considered an artifact of the sample spacing rather than an indication that more recent variations have occurred at a lower frequency.

\section{EOLIAN DEPOSITION IN THE SOUTHEAST PACIFIC}

The sediments recovered during Leg 92 were a mixture of nannofossil ooze and clay. Small numbers of foraminifers, and small amounts of zeolites, volcanic glass, palagonite, and opaque grains were also found in the sediment (site chapters, this volume). The sediments ranged in age from late Oligocene (28.5 Ma) to Pleistocene, with the mass accumulation rates of the bulk sediment varying from a low of $5 \mathrm{mg} /\left(\mathrm{cm}^{2} \times 10^{3} \mathrm{yr}\right.$.) to a high of $1125 \mathrm{mg} /\left(\mathrm{cm}^{2} \times 10^{3} \mathrm{yr}\right.$ ) (the latter occurring in the reworked sections of Hole 599). The MAR of the eolian component is quite low, averaging between 0.13 and $5.0 \mathrm{mg} /\left(\mathrm{cm}^{2} \times 10^{3}\right.$ yr.) (Table 1$)$.

\section{Hole 597}

Two lithologic units occur at Hole 597. Unit I ( 0 to $1.4 \mathrm{~m}$ sub-bottom) is from Pleistocene to middle Miocene in age and consists mainly of zeolitic clay. Unit II (1.4 to $48.4 \mathrm{~m}$ sub-bottom) is composed of clay-bearing nannofossil ooze and ranges in age from middle Miocene to late Oligocene. The mass accumulation rate of the eolian component was calculated for each sample (Table 1), and several fluctuations were observed (Fig. 2A). The eolian MAR for Hole 597 has been extremely low for the past $17 \mathrm{~m} . \mathrm{y}$., generally less than $2 \mathrm{mg} /\left(\mathrm{cm}^{2} \times\right.$ $10^{3} \mathrm{yr}$.). Between 17 and $19 \mathrm{Ma}$, eolian MAR averages 8 $\mathrm{mg} /\left(\mathrm{cm}^{2} \times 10^{3}\right.$ yr. $)$. From early Miocene to late Oligocene, the MAR is again low, ranging from 1 to $5 \mathrm{mg}$ / $\left(\mathrm{cm}^{2} \times 10^{3} \mathrm{yr}\right.$.); basal sediments contain a greater amount of mineral material (Table 1, Fig. 2A). The median grain size of the eolian component averages about $8.1 \phi$ in sediments of Unit II (Table 1, Fig. 2B).

\section{Hole 598}

The sediments in Hole 598 comprise two lithologic units, which range in age from Pleistocene to early Miocene. Unit I ( 0 to $44.6 \mathrm{~m}$ sub-bottom) is clay-bearing to clayey nannofossil ooze, with trace amounts of foraminifers, volcanic glass, palagonite, zeolites, micronodules, and opaques. Unit II ( 44.6 to $52.4 \mathrm{~m}$ sub-bottom) consists of yellowish brown clay and foraminifer-bearing nannofossil chalk.

The MAR of the eolian component from the Pleistocene to the early Miocene is again surprisingly low and constant at about $3 \mathrm{mg} /\left(\mathrm{cm}^{2} \times 10^{3} \mathrm{yr}\right.$. $)$, with a peak at $8.2 \mathrm{Ma}$ (Fig. 3A, Table 1). This increase may be a result of the volcanic glass remaining in the processed samples after extraction.
The eolian grain size data from this site exhibit the only distinct change in grain size observed in the Leg 92 sequences (Fig. 3B). The grain size of dust from sediment younger than $9.5 \mathrm{Ma}$ is coarser, with an average size of $7.2 \phi$. The lower sediments at this site (those aged from 11.8 to $16 \mathrm{Ma}$ ) have an average eolian grain size of $8.2 \phi$; the equivalent change in grain mass is an increase by a factor of 8 .

\section{Hole 599}

Hole 599 provides a record of late Miocene to Pleistocene sedimentation. This site consists of one lithologic unit of clayey and clay-bearing nannofossil ooze. The late Miocene sediments (11.9 to $35 \mathrm{~m}$ sub-bottom) have been extensively reworked (Site 599 chapter, this volume; Knüttel, this volume; Rea and Janecek, this volume).

The MAR of the eolian component (Table 1, Fig. 4A) is again low from the Pliocene to Recent, averaging $2 \mathrm{mg} /\left(\mathrm{cm}^{2} \times 10^{3} \mathrm{yr}.\right)$. Near the Pliocene/Miocene boundary, the MAR increases noticeably from 3 to $9 \mathrm{mg} /\left(\mathrm{cm}^{2} \times\right.$ $10^{3} \mathrm{yr}$.), and it increases again between Samples 599-3-5 (101-104 cm) and 599-4-3 (101-104 cm). These samples occur at depths characterized by sediment reworking that occurred during nannofossil Zone CN9b (between 5.4 and 6.7 Ma; Knüttel, this volume). However, an examination of the smear slides of the samples from the interval shown with dashed lines shows that the samples were contaminated and not thoroughly rinsed during the extraction procedure, so the observed MAR maxima from 6 to 7 Ma may be the result of improper extraction as well as ocean floor reworking. The MAR values for the earlier part of the late Miocene are again low, averaging $2 \mathrm{mg} /\left(\mathrm{cm}^{2} \times 10^{3} \mathrm{yr}\right.$.), if the lowermost sample (599-4-6, $51-54 \mathrm{~cm})$ is excluded.

Median eolian grain size (Table 1, Fig. 4B) exhibits large variations throughout the section, ranging from 8.5 to $6.5 \phi$. We believe the variations to be in part the result of volcanic glass still present in the samples and point out that no major shifts in grain size occur. In average size (about $7.3 \phi$ ), these grains are not statistically different from those in the upper portion of Hole 598.

\section{Hole 600C}

Hole $600 \mathrm{C}$, drilled in very thin sediment cover, recovered two lithologic units. Unit I ( 0 to $10 \mathrm{~m}$ sub-bottom) is composed of clay-bearing to clayey nannofossil ooze. Unit II $(10$ to $11.8 \mathrm{~m})$ consists of dark brown foraminifer-bearing clayey nannofossil ooze.

The MAR of the eolian component is consistent with that of the other sites, and little variation is observed (Table 1, Fig. 5A). The average MAR is $1.0 \mathrm{mg} /\left(\mathrm{cm}^{2} \times\right.$ $10^{3} \mathrm{yr}$.), and median eolian grain size averages about $7.7 \phi$ (Fig. 5B).

\section{Hole 601}

Hole 601 was drilled $1.85 \mathrm{~km}$ from Hole $600 \mathrm{C}$, and the sediments were divided into two lithologic units: Unit I, 0.0 to $20.0 \mathrm{~m}$ sub-bottom, and Unit II, 20.0 to $20.4 \mathrm{~m}$ sub-bottom. The sediments consisted of clay-bearing and 

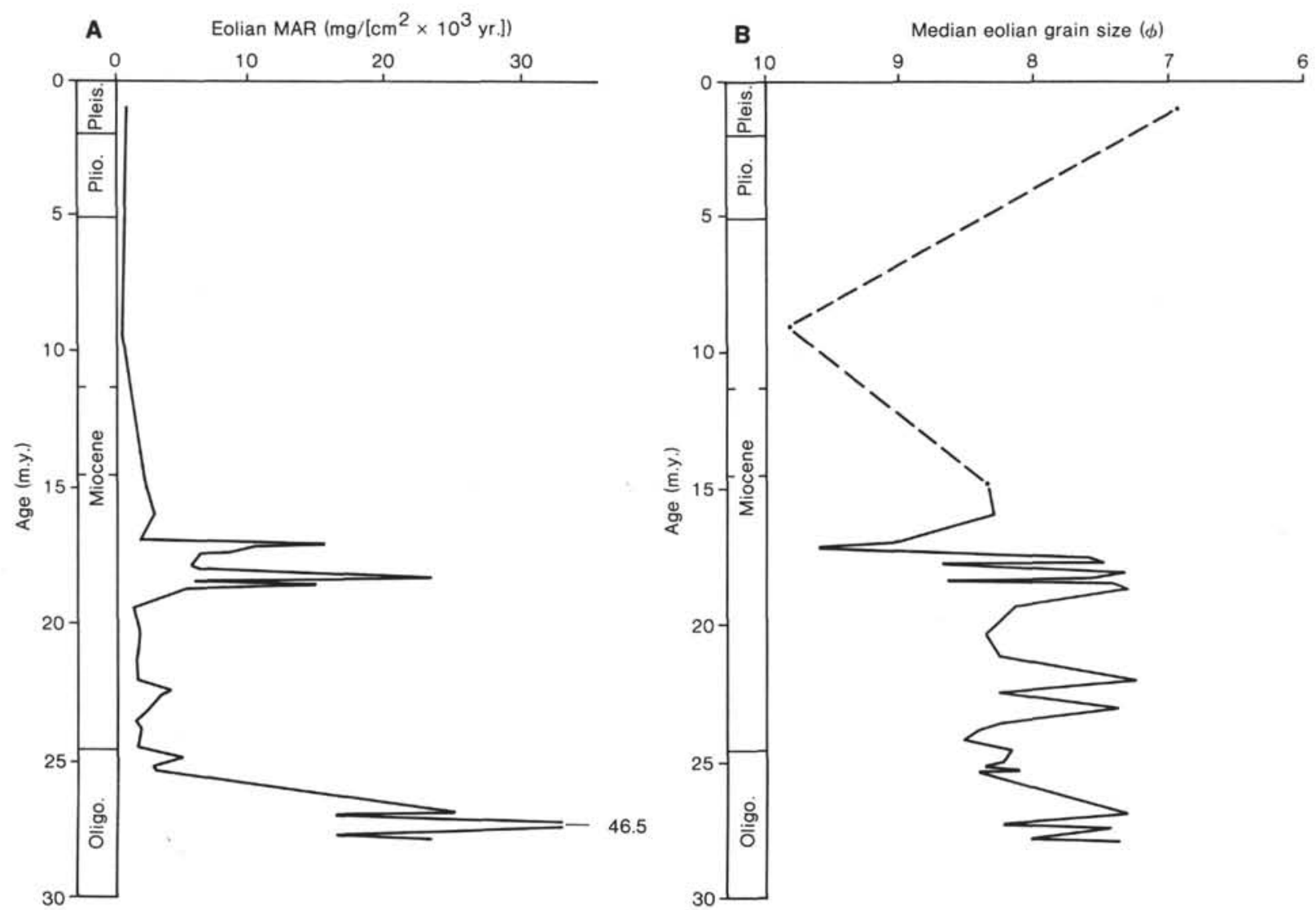

Figure 2. A. Mass accumulation rate of the eolian component of deep-sea sediments from Hole 597. Extreme values are not plotted (see Table 1). B. Eolian grain size for Hole 597.

clayey nannofossil ooze, foraminifer-nannofossil clay, and clayey nannofossil chalk. MAR for the eolian component (Table 1 , Fig. 5A) is 1 to $2 \mathrm{mg} /\left(\mathrm{cm}^{2} \times 10^{3} \mathrm{yr}\right.$.). The median eolian grain size has no observable change through the core and averages $7.5 \phi$ (Fig. 5B).

\section{IMPLICATIONS OF THE EOLIAN RECORD}

The record of the accumulation rate and grain size changes of eolian material in the East Pacific is far from precise but still allows us to draw some important conclusions. We did not find, as we expected, that the major circulation and climatic changes recorded by similar data from several sites in the North Pacific (Rea and Janecek, 1982; Janecek and Rea, 1983) were also evident in the South Pacific Leg 92 cores. The MAR of the eolian sediments is consistently low throughout the cores (Table 1). Grain size varies widely between samples, and we hesitate to attach paleoclimatic significance to the variations for the reasons previously mentioned (largely the presence of volcanic glass in the samples). The only real shift in grain size occurs in Hole 598, where eolian dust younger than about $10.5 \mathrm{Ma}$ is significantly coarser than older grains.

Previous studies of the Cenozoic suggest that climatic variations occurred during discrete periods of rapid change (Savin, 1977; Schnitker, 1980; Kennett, 1982). These periods coincide with periods of polar cooling and ice buildup, which in turn affected the pole-to-equator temperature gradient and the vigor of zonal wind patterns. The history of polar cooling is recorded by increases in the grain size of the eolian component at several North Pacific cores (Rea and Janecek, 1982; Janecek and Rea, 1983, Rea et al., 1985). We interpret this information and the eolian MAR and grain size data from the Leg 92 holes as follows.

The most striking characteristic of the influx of eolian dust to the deep-sea Leg 92 sediments is that it has been constant and low during the past 28.5 m.y. By comparison, the overall influx of eolian dust to several sites in the Northern Hemisphere has been 1 to 2 orders of magnitude larger. In terms of variation, the eolian MAR values in the South Pacific do not increase in response to either the development of the Antarctic ice cap about 12 to $13 \mathrm{Ma}$ or the onset of Northern Hemisphere glaciation $2.5 \mathrm{Ma}$. A few peaks in the MAR do occur, but at Site 599 they correspond to periods of sediment reworking and possibly the laboratory contamination of samples, and at Site 597 and possibly 598 they correspond to samples in which volcanic glass remained after the extraction process. 


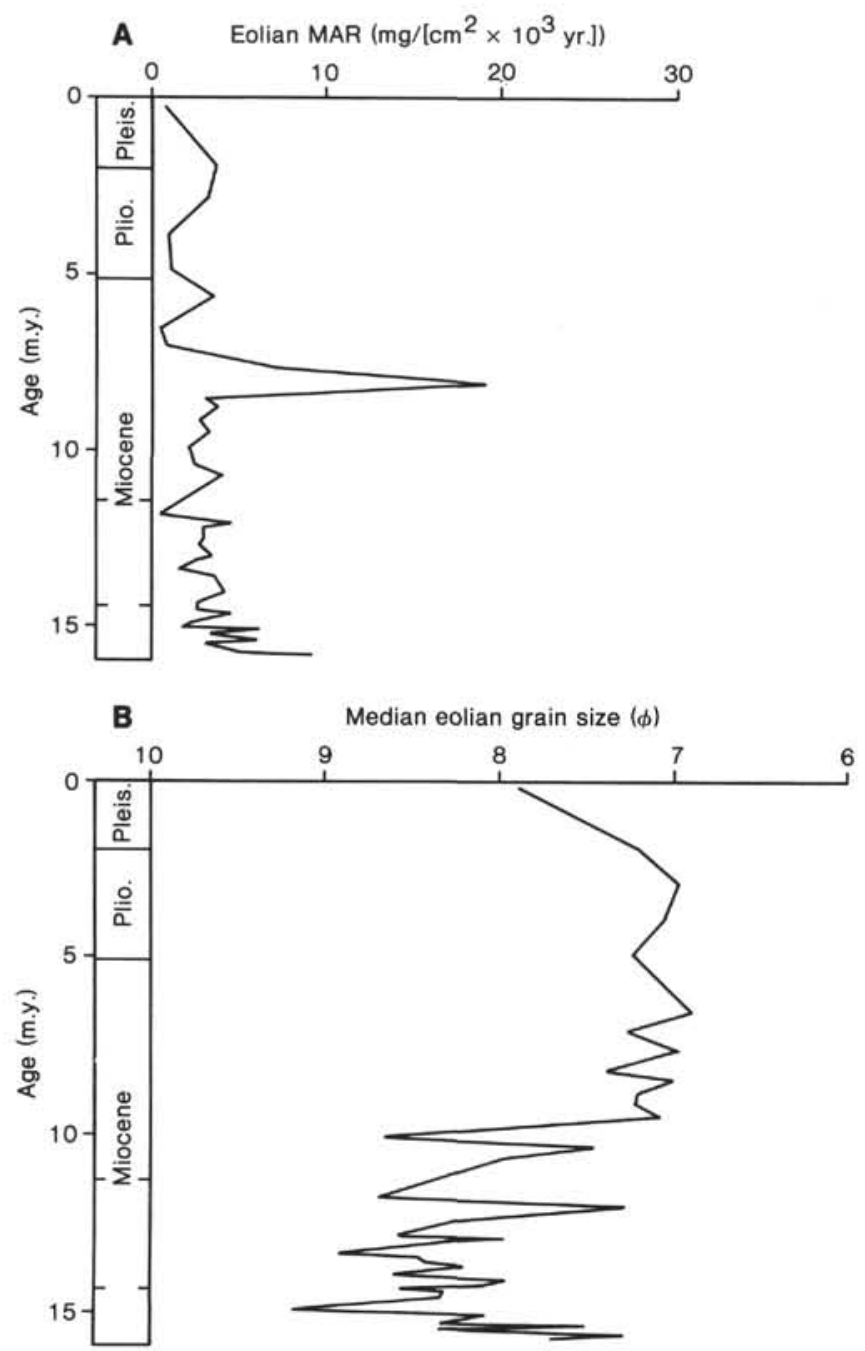

Figure 3. A. Mass accumulation rate of the eolian component of deepsea sediments from Hole 598. B. Eolian grain size for Hole 598.

In terms of changes in eolian grain size, only a single primary shift occurs in the data from Sites 597 through 601: at Hole 598, between 11.8 and $9.5 \mathrm{Ma}$, average grain size changes from $8.2 \phi$ in older sediments to $7.2 \phi$ in the younger sediments. Unfortunately, the grain size change at about 10.5 Ma is recorded neither at Hole 599, where basement age is about $8 \mathrm{Ma}$, nor at Hole 597, where this interval is represented by slowly accumulating pelagic clay and is therefore essentially unsampled at our 1-m sampling interval. We note, however, that the data from these holes are consistent with the record from Hole 598 (Figs. 2B, 3B, and 4B): the average eolian grain size of the upper Oligocene to lower Miocene materials in Hole 597 is $8.1 \phi$, and the average eolian grain size from the upper Miocene to Pleistocene sediment in Hole 599 is about $7.3 \phi$.

This shift in size may result from the 12- to 13-Ma development of the Antarctic ice cap, which increased the intensity of atmospheric circulation, as recorded in cores from the Northern Hemisphere (Rea et al., 1985). Work by Woodruff et al. (1981) on the oxygen-isotope values of foraminifers indicates that the onset of polar ice accumulation corresponds to foraminifer Zones N10 through

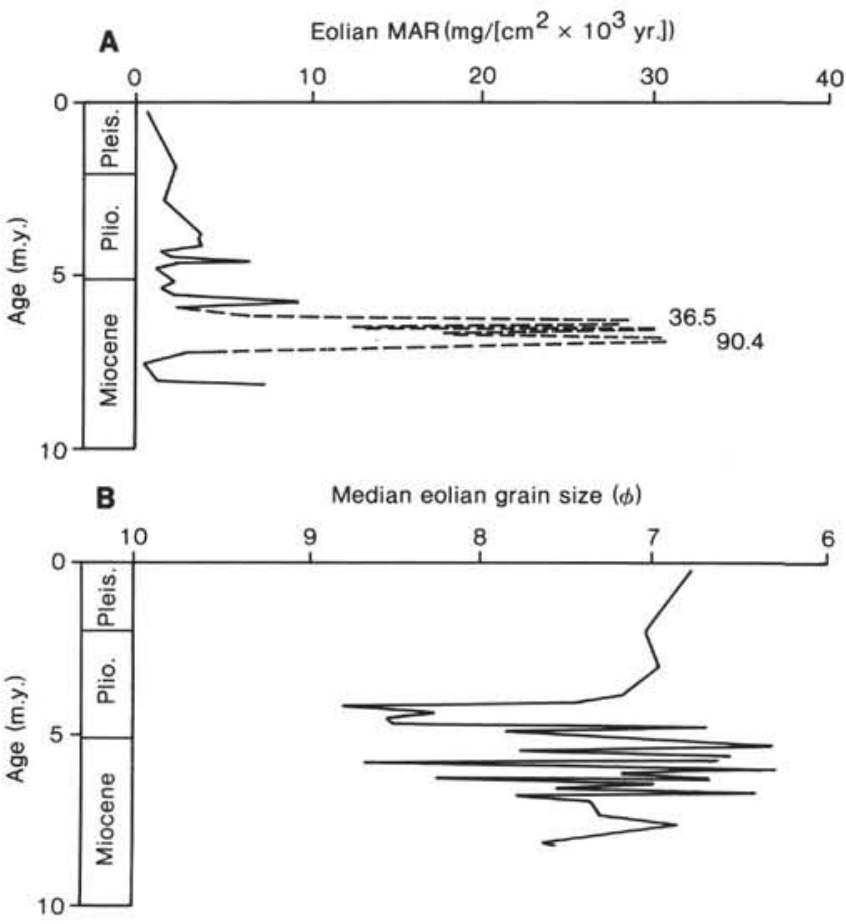

Figure 4. A. Mass accumulation rate of the eolian component of deepsea sediments from Hole 599. High values (indicated by dashed lines) may be caused by laboratory contamination. B. Eolian grain size for Hole 599.

N12; our data suggest that the change in the zonal winds occurred later (foraminifer Zones N14 and N15).

Our results have also shown that the eolian dust from deep-sea sediments in the South Pacific is, on the average, $0.5 \phi$ coarser in size than dust of equivalent age in the North Pacific (Rea and Janecek, 1982; Rea et al., 1985). The record of Antarctic glaciation which began $38 \mathrm{Ma}$ (Kennett, 1982) suggests that the South Pole has been colder than the North Pole since then and, thus, that the Southern Hemisphere's zonal winds have been more intense. Our data support this concept of hemispherical asymmetry in atmospheric circulation.

\section{CONCLUSIONS}

Isolation and quantification of the eolian component of pelagic sediments from the East Pacific Rise at $19^{\circ} \mathrm{S}$ have enabled us to develop an interpretation of the eolian processes since the late Oligocene. Our initial assumption, that the major changes in eolian deposition recorded in the North Pacific would also be evident in the South Pacific, was not confirmed. The depositional record from the Leg 92 drill sites indicates a constant and low flux of dust to the South Pacific. This suggests that South America has always been vegetated and never semi-arid, and also that orographic precipitation on the east flank of the Andes has been removing dust from the southeast trade winds throughout the timespan represented by our cores. The eolian grain size is consistently coarser than in the Northern Hemisphere, with the only major change occurring between 11.8 and $9.5 \mathrm{Ma}$. These results indicate that atmospheric circulation in the Southern Hemisphere was more intense than and acted 


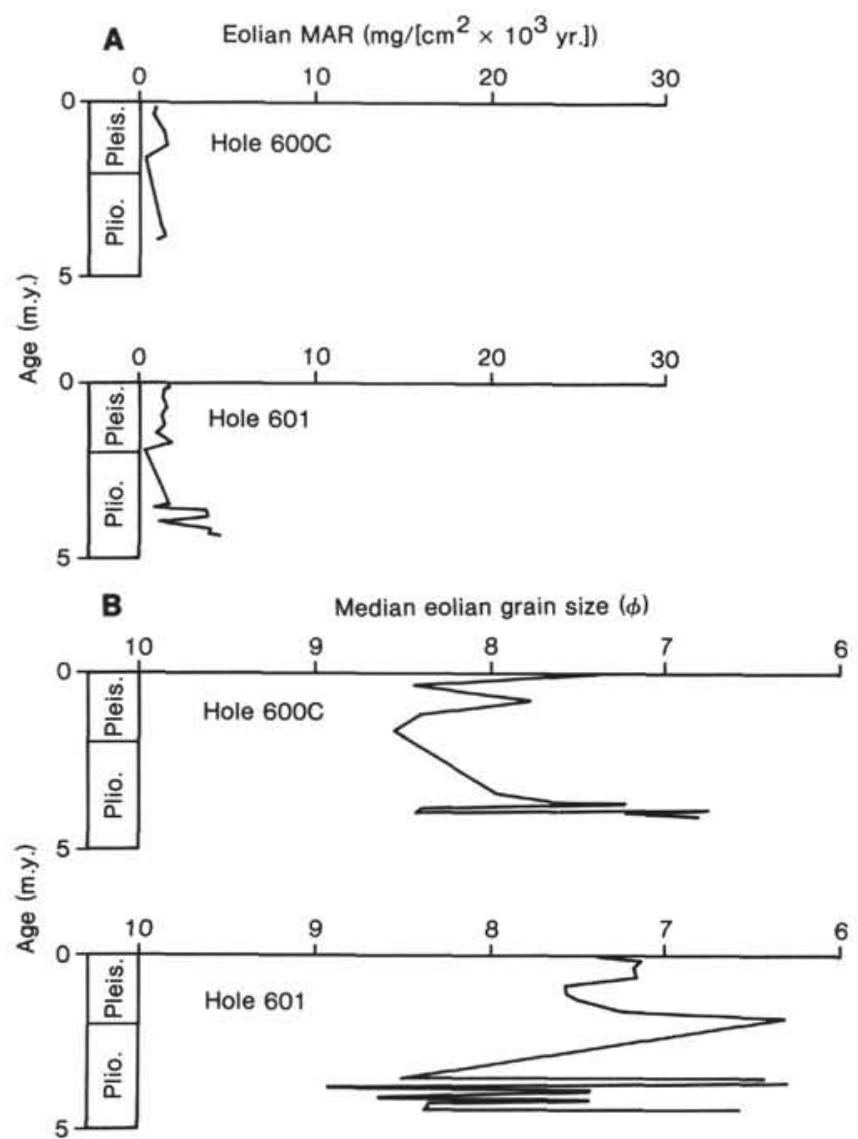

Figure 5. A. Mass accumulation rate of the eolian component of deepsea sediments from Holes $600 \mathrm{C}$ and 601 . Extreme values are not plotted (see Table 1). B. Eolian grain size for Holes $600 \mathrm{C}$ and 601.

independently of that in the Northern Hemisphere, responding differently to the various polar cooling events.

\section{ACKNOWLEDGMENTS}

We thank Nicklas Pisias and Tom Janacek for a careful review of this manuscript.

\section{REFERENCES}

Climap Project Members, 1976. The surface of the ice-age earth. Science, 191:1311-1317.

1981. Seasonal reconstructions of the earth's surface at the last glacial maximum. Map Chart Ser. Geol. Soc. Am., MC-36: Boulder, CO.

Ferguson, W. S., Griffin, J. J., and Goldberg, E. D., 1970. Atmospheric dusts from the North Pacific-a short note on long-range eolian transport. J. Geophys. Res., 75:1137-1139.

Folk, R. L., 1974. Petrology of Sedimentary Rocks: Austin, TX (Hemphill Publishing Co.).

Gates, W. L., 1976. Modelling the ice age climate. Science, 191:11381144.

Gillette, D. A., 1974. On the production of soil wind erosion aerosols having the potential for long range transport. J. Rech. Atmos., 8: 735-744.

1981. Production of dust that may be carried great distances. In Pewe, T. L. (Ed.), Desert Dust: Origin, Characteristics and Effect on Man. Spec. Paper Geol. Soc. Am., 186:11-26.

Gillette, D. A., and Walker, T., 1977. Characteristics of airborne particles produced by wind erosion of sandy soils, high plains of west Texas. Soil Sci., 12:97-110.
Glaccum, R. A., and Prospero, J. M., 1980. Saharan aerosols over the tropical North Atlantic-mineralogy. Mar. Geol., 37:295-321.

Ingle, J. C., Jr., 1973. Summary comments on Neogene biostratigraphy, physical stratigraphy, and paleo-oceanography in the marginal northeastern Pacific Ocean. In Kulm, L. D., von Huene, R., et al., Init. Repts. DSDP, 18: Washington (U.S. Govt. Printing Office), 949-960.

Jackson, M. L., Gillette, D. A., Danielsen, E. F., Blifford, I. H., Bryson, R. A., and Syers, J. K., 1973. Global dustfall during the Quaternary as related to environments. Soil Sci., 116:135-145.

Janecek, T. R., and Rea, D. K., 1983. Eolian deposition in the northeast Pacific Ocean: Cenozoic history of atmospheric circulation. Geol. Soc. Am. Bull., 94:730-738.

Kennett, J. P., 1982. Marine Geology: Englewood Cliffs, N. J. (Prentice-Hall, Inc.)

Lamb, H. H., and Woodroffe, A., 1970. Atmospheric circulation during the last ice age. Quat. Res., 1:29-58.

Leinen, M., and Heath, G. R., 1981. Sedimentary indicators of atmospheric activity in the Northern Hemisphere during the Cenozoic. Palaeogeogr. Palaeoclimatol. Paleoecol., 36:1-21.

Manabe, S., and Hahn, D. C., 1977. Simulation of the tropical climate of the ice age. J. Geophys. Res., 82:3889-3912.

Maury, M. F., 1855. The Physical Geography of the Sea: New York (Harper Bros.).

Moore, T. C., Jr., Burckle, L. H., , Geitzenauer, K., Luz, B., MolinaCruz, A., Robertson, J. H., Sachs, H., Sancetta, C., Thiede, J., Thompson, P., and Wenkam, C., 1980. The reconstruction of seasurface temperatures in the Pacific Ocean of 18,000 B.P. Mar. Micropaleontol., 5:215-247.

Parkin, D. W., 1974. Trade winds during the glacial cycles. Proc. Roy. Soc. London Ser. A, 377:73-100.

Parkin, D. W., and Padgham, R. C., 1975. Further studies on trade winds during the glacial cycles. Proc. R. Soc. London Ser. A, 346: 245-260.

Parkin, D. W., and Shackleton, N. J., 1973. Trade winds and temperature correlations down a deep-sea core off the Saharan coast. $\mathrm{Na}$ ture, 245:455-457.

Prospero, J. M., and Bonatti, E., 1969. Continental dust in the atmosphere of the eastern equatorial Pacific. J. Geophys. Res., 74:33623371.

Radczewski, O. E., 1939. Eolian deposits in marine sediments. Spec. Publ. Soc. Econ. Paleont. Mineral., 4:496-502.

Rea, D. K., and Janecek, T. R., 1981a. Late Cretaceous history of eolian deposition in the mid-Pacific mountains, central North Pacific Ocean. Palaeogeogr. Palaeoclimatol. Palaeoecol., 36:55-67.

$1981 \mathrm{~b}$. Mass-accumulation rates of the non-authigenic inorganic crystalline (eolian) component of deep-sea sediments from the western mid-Pacific mountains, Deep Sea Drilling Project Site 463. In Thiede, J., Vallier, T. L., et al., Init. Repts. DSDP, 62: Washington (U.S. Govt. Printing Office), 653-659.

1982. Late Cenozoic changes in atmospheric circulation deduced from North Pacific eolian sediments. Mar. Geol., 49:149167.

Rea, D. K., Leinen, M., and Janecek, T. R., 1985. Geologic approach to the long-term history of atmospheric circulation. Science, 227: 721-725.

Rex, R. W., and Goldberg, E. D., 1958. Quartz contents of pelagic sediments of the Pacific Ocean. Tellus, 10:153-159.

Sarnthein, M., Theide, J., Pflaumann, U., Erlenkeuser, H., Futterer, D., Koopmann, B., Lange, H., and Seibold, E., 1982. Atmospheric and oceanic circulation patterns off northwest Africa during the past 25 million years. In von Rad, U., Hinz, K., Sarnthein, M., and Siebold, E. (Eds.), Geology of Northwest African Continental Margin: New York (Springer-Verlag), 545-604.

Savin, S. M., 1977. The history of the Earth's surface temperature during the past 100 million years. Annu. Rev. Earth Planet. Sci., 5:319-355.

Schnitker, D., 1980. Global paleoceanography and its deep water linkage to the Antarctic glaciation. Earth Science Rev., 16:16-20.

Thiede, J., 1979. Wind regimes over late Quaternary southwest Pacific Ocean. Geology, 7:259-262.

van Andel, T. H., Heath, G. R., and Moore, T. C., Jr., 1975. Cenozoic History and Paleoceanography of the Central Equatorial Pacific. Mem. Geol. Soc. Am., 143. 
Windom, H. L., 1969. Atmospheric dust records in permanent snowfields: implications to marine sedimentation. Geol. Soc. Am. Bull., 80:761-782.

1975. Eolian contributions to marine sediments. J. Sediment. Petrol., 45:520-529.

Windom, H. L., and Chamberlain, C. E., 1978. Dust-storm transport of sediments to the North Atlantic Ocean. J. Sediment. Petrol., $48: 385-388$.
Woodruff, F., Savin, S. M., and Douglas, R. G., 1981. Miocene stable isotope record: a detailed deep Pacific Ocean study and its paleoclimate implications. Science, 212:665-668.

Date of Initial Receipt: 13 July 1984 Date of Acceptance: 26 December 1984 